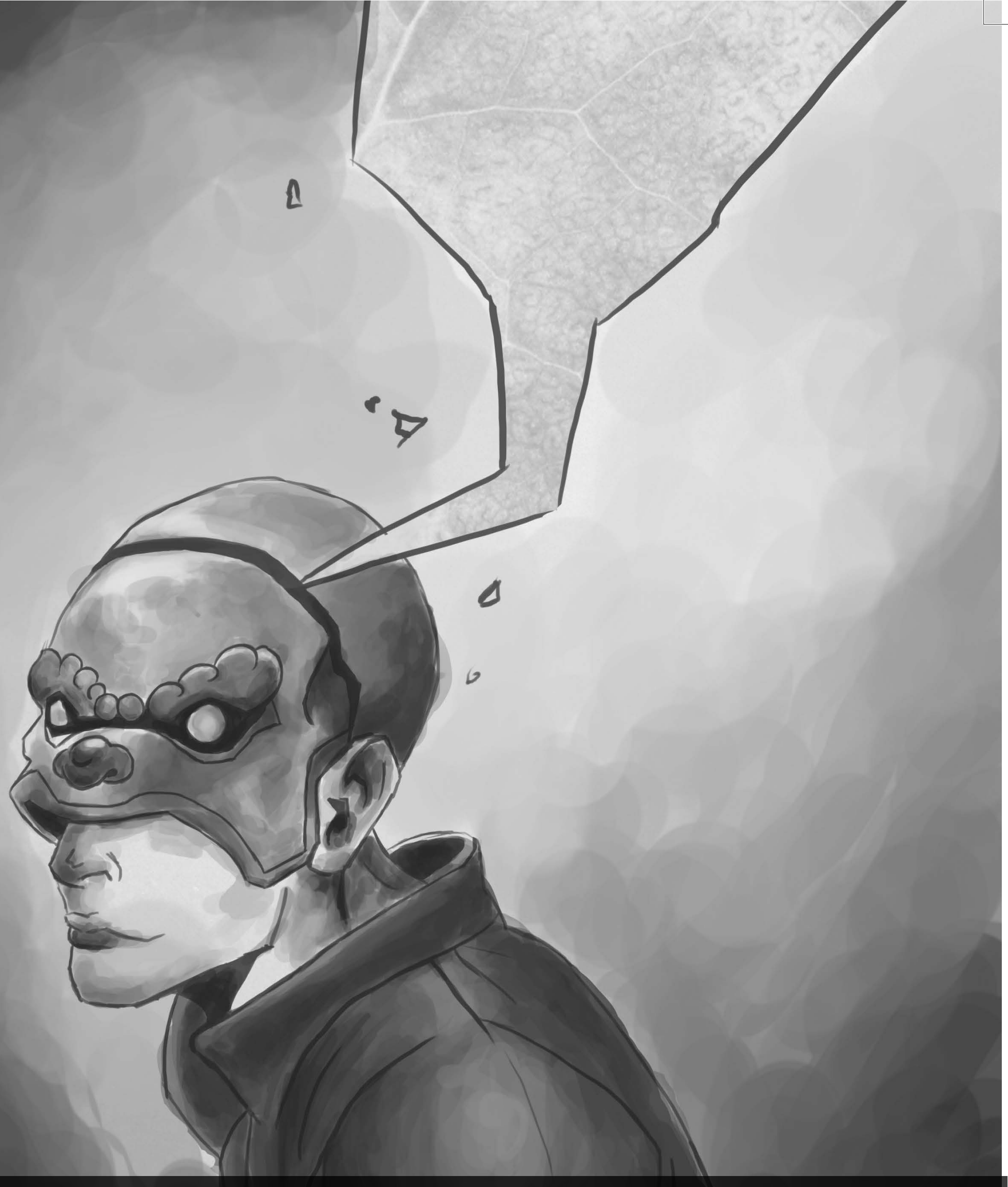

Estudio exploratorio del clima creativo en compañías colombianas seleccionadas 


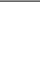




\section{Estudio exploratorio del clima creativo en compañías colombianas seleccionadas ${ }^{1}$}

Fecha de recepción: 25 de julio de 2011

Fecha de aprobación: 25 de octubre de 2011

\section{John Fitzgerald Cabra Vidales}

cabraj@@buffalostate.edu

Doctor en Psicología organizacional de la Universidad de Manchester, Inglaterra. Profesor asistente de la State University of NewYork, Buffalo

State. Facultad en el International Center for Studies in Creativity.

\section{Resumen}

Este estudio procura identificar los aspectos organizaciones que facilitan y dificultan la creatividad en el lugar de trabajo para determinar si existe algún concepto que se distingue o es relevante en una muestra de compañías colombianas seleccionadas. Fueron entrevistados 52 directores ejecutivos, gerentes, supervisores y empleados de primera línea de ocho empresas establecidas en Colombia. Se aplicó la metodología de la teoría fundamentada. El análisis arrojó similitudes y diferencias: los temas destacados fueron envida y celos, pertenencia, creatividad intencional, normas del manejo de influencia y equidad.

\section{Palabras clave}

Ambiente creativo, creatividad, desarrollo organizacional, teoría fundamentada.

\section{Juliana Sánchez Trujillo}

\section{julist24@gmail.com}

Candidata a la Maestría de Creatividad e Innovación en el Centro Internacional de Estudios en Creatividad de la State University of New York y Comunicadora social con énfasis en comunicación organizacional de la Pontificia Universidad Javeriana de Bogotá.

\section{Abstract}

This study sought to identify aspects of organizations that help and hinder workplace creativity and to determine if there are any distinctive or relevant concepts to a sample of select Colombian organizations. Fifty-two managing directors, managers, supervisors, and front-line employees from eight companies headquartered in Colombia were interviewed. Grounded Theory was applied to this study. An analysis produced distinct themes such as Envy/Jealousy, belonging, organized creativity, influence management norms, sense of equity.

$1 \quad$ Borradores anteriores y más extensos de este trabajo fueron publicados en inglés en Cuadernos de Administración. Bogotá, Colombia 29, 53-86 y presentados en español en la conferencia de la Asociación Europea de la Creatividad en Sestri Levanti, Italia.

Los autores desean agradecer a los profesores Reginald Talbot de la Univeridad de Manchester, Andrew Joniak y a Gerard J. Puccio, director del International Center for Studies in Creativity de State University of New York, Buffalo State, por sus sugerencias y comentarios. 


\section{Introducción}

El ritmo de la vida se ha incrementado de forma exponencial en las últimas décadas, transformando la forma en que los seres humanos se relacionan. Mientras que en un período de dos mil años surgieron diez inventos tecnológicos y sociales que tuvieron un impacto considerable en la historia como los sistemas de riego, el sistema numérico y el sistema monetario, en los últimos 200 años se han generado 25 inventos que han alterado de forma dramática el curso de la humanidad. Entre estas invenciones se cuentan los aviones, antibióticos, la clonación, la computación, las tarjetas de crédito, el teléfono y el Internet (Henry, 2001). Esta cadencia a la que nos vemos expuestos hoy, exige personas flexibles, ágiles y con niveles de creatividad excepcionales que les permitan competir en un mundo en el que el ciclo de los productos es muy corto y en que la adaptabilidad al cambio resulta determinante para competir en mercados globales.

En un estudio realizado por el Instituto de negocios de valor de IBM (2010), se estableció que la cualidad de liderazgo más importante en los próximos cinco años será la creatividad, pues ésta se reconoce como la materia prima de la innovación. Para promover esta habilidad, el estudio propuso el establecimiento de temas de capacitación en creatividad como elementos fundamentales para catalizar procesos de innovación. En otras palabras, las organizaciones están exigiendo altos niveles de creatividad en sus empleados, ¿pero están en realidad preparados para desarrollar en ellos habilidades de pensamiento creativo? El reconocimiento de que la ventaja estratégica competitiva puede alcanzarse a través de la creatividad y nuevos enfoques para alcanzar los objetivos organizacionales, ha generado nuevas culturas corporativas que promueven las actitudes y valores compartidos mientras promocionan la generación de nuevas ideas tanto a nivel individual como grupal (Andriopoulos y Dawson, 2009).

Los temas de creatividad e innovación se encuentran directamente relacionados. Para muchos la creatividad es el primer paso hacia la innovación, aquella fuerza que conduce al cambio. En el presente artículo, los autores proponen entender la creatividad como novedad que es útil. Esta definición proporciona un balance entre la originalidad que muchos buscan al hablar de este tema, pero al mismo tiempo enfatiza que esta singularidad debe servir a un propósito (Firestien, 2004).

En la historia muchos han definido la creatividad desde diferentes perspectivas. El investigador Mel Rhodes (1961), se dio a la tarea de encontrar una única definición del término, pero a cambio descubrió cuatro enfoques fundamentales desde los cuales se hace posible el estudio de la creatividad: persona, proceso, producto y entorno. La persona define las características de personalidad que caracterizan al individuo creativo; el proceso habla de los medios a través de los cuales las personas generan e implementan nuevas ideas; el producto intenta definir cuándo un prototipo u objeto puede considerarse creativo; finalmente el entorno (propuesto por Rhodes bajo el término Press), enfatiza el tipo de ambientes en el que una persona es más apta a generar ideas, involucrarse en la vida de la organización y hacer suyas las nuevas iniciativas que ésta pretende implementar. Estos factores se encuentran directamente relacionados y el desarrollo de uno afecta de manera considerable las funciones del otro. Para efectos de esta investigación, el enfoque será fundamentalmente en los factores que afectan el entorno creativo.

Teresa Amabile (1996) determinó que los factores que limitan la creatividad en el lugar de trabajo son la expectativa de evaluación, vigilancia, motivación extrínseca, los incentivos, la competencia y la falta de autonomía. Por su parte, la investigación realizada por Ekvall et al. (1983), planteó diez dimensiones que fomentan la creatividad en el lugar de trabajo. Ellas son: lúdica y humor, retos, libertad, soporte a las ideas, tiempo para las ideas, confianza, dinamismo, toma de riesgos, debate y bajos indices de conflicto. El entendimiento de estos factores como temas de aplicación global, han impulsado el desarrollo de esta investigación.

A medida que el ritmo de la globalización se intensifica, los administradores se enfrentan cada vez más a una variedad de valores nacionales que seguramente habrán de influir en la transferencia de los sistemas de manejo (Schneider, 1988) de las culturas occidentales hacia las latinoamericanas. Tales circunstancias los instan a orientar a las personas durante los periodos de variaciones considerables, enmarcados por la confusión y los cambios indefinidos en el entorno. Pero los entornos nebulosos crean también oportunidades para 
la creatividad (Bridges, 1991). De alli que la habilidad para ser creativos se torna crítica si una compañía le apunta a las necesidades globales para asegurar su supervivencia en los países objeto. Por lo tanto, más conocimiento y más esfuerzo deliberado son necesarios para considerar la unicidad de países latinos. Este artículo propone un estudio sobre los aspectos organizacionales que soportan $\mathrm{u}$ obstaculizan la creatividad en compañias colombianas seleccionadas.

Los investigadores usaron la metodología empleada por Burnside et al. (1998) y Ekvall et al. (1983) como base del estudio. Al parecer son pocos los investigadores que directamente obtuvieron muestras a través de entrevistas semiestructuradas, lo que ayuda y obstaculiza la creatividad en el sitio de trabajo en países en América Latina. Sin embargo, en el estudio de Burnside et al. (1998), se utilizó el personal de R\&D (Investigación y Desarrollo) porque representaba un área usualmente encargada de la parte creativa (Burnside, et al 1988). En este estudio, la gente de R\&D no representaba la población. Las otras metodologias como la revisión de la literatura o las traducciones de cuestionarios establecidos no eran atractivas porque no garantizaban que un estudio cualitativo per se produjera temas distintivos al momento de transferir la investigación a otros países. La metodología de Burnside et al. (1988) y de Ekvall et al. (1983) genera una cantidad de información más útil y más expansible; de allí que, dada la naturaleza nacional de este estudio, esta metodología es la más apropiada.
Por tal motivo, el objetivo del presente estudio es explorar factores que facilitan u obstaculizan la creatividad en el sitio de trabajo para determinar si hay algún concepto que sea único para la muestra colombiana que potencialmente amerite su inclusión en un cuestionario sobre el clima laboral. Esto es importante porque existe la necesidad de garantizarle a los usuarios de estos cuestionarios, fundamentos teóricos significativos y representativos dentro de la cultura objeto. También es importante porque existe la posibilidad de que los investigadores diseminen información que no represente ni entienda a los grupos de otras culturas (Valencia, 2000). Por lo tanto, este estudio es un primer paso hacia la construcción de una medición donde se incluyen análisis con datos cualitativos.

\section{Muestra}

\section{Características organizacionales}

Al seleccionar las organizaciones para el estudio, el criterio principal era que fueran compañias nacionales de 150 o más empleados. Una compañia como American Airlines no sería útil porque tiene su base de acción en los Estados Unidos y es posible que imponga su cultura corporativa y operacional sobre sus estaciones de servicios internacionales. La Tabla 1 presenta un análisis detallado de un muestreo de frecuencias por nivel y de acuerdo con otros datos demográficos.

TABLA 1 MUESTRA DEMOGRÁFICA

\begin{tabular}{|c|c|c|c|c|c|c|c|c|c|c|}
\hline & $\begin{array}{l}\text { Manuf. } \\
\text { electric. } \\
\text { transf. }\end{array}$ & $\begin{array}{l}\text { Ingenio } \\
\text { de } \\
\text { azúcar }\end{array}$ & $\begin{array}{l}\text { Supermerc, } \\
\text { medicina, y } \\
\text { servicios de } \\
\text { recreación }\end{array}$ & $\begin{array}{l}\text { Cadena } \\
\text { de } \\
\text { comida } \\
\text { rapida }\end{array}$ & $\begin{array}{l}\text { Banco } \\
\text { Nacional }\end{array}$ & $\begin{array}{l}\text { Procesadora } \\
\text { productos } \\
\text { avicolas }\end{array}$ & $\begin{array}{l}\text { Tele- } \\
\text { Comm. }\end{array}$ & $\begin{array}{l}\text { Empresa } \\
\text { Electrica }\end{array}$ & Otra & Total \\
\hline Sitio & $\mathrm{HDQ}$ & $\mathrm{HDQ}$ & $\mathrm{HDQ}$ & $\mathrm{HDQ}$ & $B / R$ & $\mathrm{HDQ}$ & $\mathrm{B} / \mathrm{R}$ & $H D Q$ & & \\
\hline $\begin{array}{l}\text { \# de } \\
\text { Empleados }\end{array}$ & 150 & 1100 & 1300 & 1200 & 2500 & 860 & 6500 & 180 & & \\
\hline$\%$ de Mercado & $72 \%$ & $6.26 \%$ & - & - & $12 \%$ & $30 \%$ & - & - & & \\
\hline Directores & 0 & 0 & 0 & 3 & 0 & 0 & 1 & 0 & 1 & 5 \\
\hline Gerentes & 2 & 3 & 4 & 3 & 2 & 2 & 3 & 2 & 0 & 21 \\
\hline Supervisores & 0 & 0 & 0 & 1 & 2 & 2 & 0 & 4 & 0 & 9 \\
\hline
\end{tabular}




\begin{tabular}{lcccccccc}
\hline & $\begin{array}{l}\text { Manuf. } \\
\text { electric. } \\
\text { transf. }\end{array}$ & $\begin{array}{l}\text { Ingenio } \\
\text { de } \\
\text { azúcar }\end{array}$ & $\begin{array}{l}\text { Supermerc, } \\
\text { medicina, } \\
\text { servicios de } \\
\text { recreación }\end{array}$ & $\begin{array}{l}\text { Cadena } \\
\text { de } \\
\text { comida } \\
\text { rapida }\end{array}$ & $\begin{array}{l}\text { Banco } \\
\text { Nacional }\end{array}$ & $\begin{array}{l}\text { Procesadora } \\
\text { productos } \\
\text { avicolas }\end{array}$ & $\begin{array}{l}\text { Tele- } \\
\text { Comm. }\end{array}$ \\
\hline Obrempresa \\
Electrica \\
Otra
\end{tabular}

* Nota: $\quad H D Q$ refiere a la casa matriz. B/R refiere a la oficina regional

Siendo fiel a ese criterio, se seleccionaron las empresas que se listan a continuación:

-Compañía fabricante de transformadores eléctricos con base en la ciudad de Pereira.

-Refinería de azúcar con base en la ciudad de Buga.

-Proveedor de abarrotes, servicios médicos y recreativos para los empleados de empresas-clientes y para el público en general con operaciones centrales en Pereira.

-Cadena de restaurantes de comidas rápidas que se especializa en pollo frito y que tiene su base empresarial en Dosquebradas.

-Banco nacional. Éste tiene su base en Medellín, pero los entrevistados son empleados de la sucursal de Pereira.

-Empresa procesadora de productos avícolas que además administra criaderos de pollo; sus oficinas principales están situadas en Pereira.

-Empresa de telecomunicaciones operada por el gobierno colombiano (Esta compañia fue liquidada en junio del año 2000 poco después de haber terminado las entrevistas). Sin embargo, los entrevistados eran empleados en la oficina regional de
Pereira y no de sus instalaciones de Bogotá.

-Proveedor de servicios públicos en Pereira, operada por el gobierno local.

Jorgensen, Hafsi, y Kiggundu (1986) desarrollaron un esquema organizacional que identifica a las organizaciones usualmente encontradas en los países en desarrollo. Son empresas de propiedad gubernamental y estatal, organizaciones de propiedad familiar, subsidiarias multinacionales e industriales. Las organizaciones que fueron involucradas en este estudio representaban a cada grupo de acuerdo con la taxonomía de Jorgensen et al. (1986). El banco nacional hace parte del grupo multinacional con oficinas en Estados Unidos, Panamá y las Islas Caimanes. Los proveedores de telecomunicaciones y servicios públicos hacen parte del grupo de propiedad gubernamental y estatal. La cadena de restaurantes hace parte del grupo de propiedad familiar. La empresa avícola, el conglomerado de abarrotes y la fábrica de transformadores eléctricos hacen parte del grupo industrial. La refinería de azúcar se sale de esta taxonomía y representa a la industria agrícola.

\section{Características de los participantes}

Cincuenta y dos participantes de ocho compañias participaron en este estudio. La muestra restante $(n=9)$ fueron personas conocidas y contactos personales en Colombia. Los entrevistados fueron divididos en tres tipos diferentes de informantes para componer una triangulación de datos (Arksey y Knight, 1999): informantes de compañias objetivo $(n=52)$, profesores universitarios $(n=5)$ y empleados de compañias no objetivo $(n=4)$. La muestra de compañías no objetivo constaba de un 
consultor empresarial, un director de una incubadora de ideas empresariales, un trabajador de alto rango empleado por una fábrica de yogurt y otros productos lácteos, y un trabajador de alto nivel empleado por una empresa fabricante de textiles. Miles y Huberman (1994) sugirieron el uso de estos tipos de contactos para enriquecer las perspectivas culturales, examinar la profundidad de algunos comentarios de los entrevistados (por ejemplo: bajos salarios y la carencia de participación en utilidades, lo cual desestimula la creatividad), y validar la información recibida (Arksey, y Knight, 1999). Los datos fueron recolectados entre julio y octubre del año 2000.

\section{Metodología}

\section{Procedimiento de reclutamiento}

En este estudio, las entrevistas individuales fueron seleccionadas de tal manera que no comprometiera la privacidad y confidencialidad. El investigador determinó que las entrevistas individuales podrían con toda probabilidad reducir la ansiedad del participante, causada por el miedo a las repercusiones que podria tener la posibilidad de sacar a la luz pública material confidencial tal como factores del clima laboral que actúan como barreras de la creatividad. Una técnica de incidente crítico fue utilizada para identificar los comportamientos y factores que contribuyen al éxito o fracaso de los individuos y organizaciones en situaciones especificas (Andersson y Nilsson, 1964). Los investigadores solicitaron sus propios ejemplos de creatividad. Éstos no tenían que ser los experimentados directamente por ellos, sino que podían incluir un evento vivido por un colega de trabajo. Las preguntas principales fueron tomadas y adaptadas de Amabile y Gryskewicz (1988) y fueron las siguientes:

¿Cuál fue el evento, la idea, producto o proceso creativo?

¿Cuál fue la circunstancia?

¿Quién de la compañía facilitó este evento creativo?

¿Cómo facilitaron el evento creativo?
¿Qué características o habilidades de la gente involucrada contribuyeron a la creatividad del evento?

¿Qué aspectos de la compañía facilitaron el acto creativo?

¿De qué manera facilitaron estos aspectos el acto creativo?

¿Fueron superados algunos obstáculos en el entorno laboral en el proceso?

¿Quién de la empresa obstaculizó el acto creativo?

¿De qué manera obstaculizaron el acto creativo?

¿De qué manera bloquean estas cosas el acto creativo?

\section{Codificación}

La fase de codificación comenzó a usarse como enfoque teórico fundamentado. Los fundamentos teóricos son un enfoque interpretativo para estudiar los datos cualitativos. En esencia, la teoria se basa en los datos que emergieron de este estudio. Por ejemplo, las categorias del clima creativo derivadas de otras culturas no deben asumirse como aplicables universalmente a Colombia. Tal vez se puedan identificar algunas categorias que son específicas para las culturas latinoamericanas. Puesto que las categorias pueden ser parcialmente indeterminadas, un enfoque de base teórica fue el más apropiado.

La teoría fundamentada comienza cuando los investigadores se "sumergen" en las trascripciones y otros documentos para identificar temas importantes (Abrahamson, 1983). Esta teoría involucra el examen de datos donde los investigadores se encuentran inmersos en el texto para descubrir temas y conceptos que parecen importantes para los creadores de cada comentario (Strauss y Corbin, 1990; Berg, 2001). Con ese fin, se utilizó un proceso de codificación narrativo a tres niveles, el cual es descrito con más detalle en una obra doctoral (Cabra, 2006). 


\section{Resultados}

El método de la teoría fundamentada identificó 16 categorías desde las 606,852 narrativas codificadas y comprendió aproximadamente el $75 \%$ del texto. Las narrativas no codificadas o no estaban relacionadas con el clima creativo ni contenian unidades significativas de información, o estaban relacionadas con el clima creativo, pero no aparecían con la frecuencia suficiente como para formar una categoría. Traición (1), irrespeto por el colega (1), comportamiento antagonista (1), insatisfacción por la escritura y elaboración de ideas (1), debate (1), repetición de errores (1), paciencia (1), comportamiento anarquista (1), mentiras piadosas (2), demasiada sensibilidad (2), conflicto (2), integridad (3), ética (3), autoconciencia (3), tacto (3), mentalidad de unión negativa (3), excusas (3), mal genio (3), bajo nivel de conciencia social (4), y seguridad laboral (4). Las 16 categorías están detalladas aquí.

\section{Recursos}

El entrenamiento, dinero, tecnología, facilidades, equipo e información que están siempre disponibles para que los miembros de la organización desarrollen sus ideas (Amabile, 1996; Kanter, 1984; Van Gundy, 1987). Cuando los recursos son escasos, los presupuestos son bajos y las iniciativas de recorte en los costos son comunes y constantes, este proceso se ve afectado.

\section{Confiabilidad}

La percepción de que las personas en la organización son confiables. Es decir que se puede contar con que ellos demostrarán constantemente comportamientos honestos y cabales (Ekvall, 1996; Isaksen, et al. 1999). Existe fe, por parte de la organización, de que las personas tienen la destreza, habilidad, honestidad y conducta necesarias para completar las funciones a su cargo. Cuando la confianza es poca las personas no delegan, no aceptan responsabilidades adicionales y no cumplen las promesas.

\section{Velocidad de reacción}

La capacidad organizativa de responder rápidamente a las ideas de sus miembros. En ambientes como éstos, la organización acelera en forma activa el procesamiento de ideas y se toman un sinnúmero de medidas para asegurar que una idea es implementada tan pronto como sea posible. En ambientes diferentes la centralización organizativa, la burocracia, la falta de visión politica y el interminable papeleo retardan el desarrollo de ideas (Amabile, 1996).

\section{Estilo de liderazgo}

La forma en que los líderes ejercitan el poder (Siegel y Kaemmerer, 1978). Los ambientes más aptos para la creatividad son en los que las personas se sienten honradas y respetadas, la gente propone ideas, opiniones o detecta problemas sin miedo a ser menospreciada (Lee, 1997). Por otro lado, el liderazgo no conduce a la creatividad cuando el poder y el miedo son usados como instrumentos para forzar a la gente a cumplir sin cuestionar, cuando se les maltrata con la intención de hacerlos sentir inferiores, desmoralizados e inseguros sobre sus trabajos. También cuando se les amenaza, humilla e intimida.

\section{Libertad}

Un lugar de trabajo que no es limitante, que permite que los empleados experimenten y apliquen nuevas ideas. Las personas tienen libertad y autonomía para generar ideas, desarrollarlas y tomar riesgos por su propia cuenta, sin interrupciones de la organización para controlar procedimientos y resultados. Como consecuencia, las personas se sienten libres para ser ellas mismas, sin limitaciones. Cuando no existe libertad y autonomía, las personas no son capaces de hacer elecciones o tomar decisiones por sí mismas y como resultado vacilan ante la posibilidad de actuar fuera de los límites establecidos (Burnside et al., 1988; Isaksen, et al., 1999).

\section{Sinergia}

La colaboración y unidad que existen cuando las personas trabajan juntas y unen diferentes perspectivas para alcanzar objetivos comunes (Thomas y Kilmann, 1974; Siegel y Kaemmerer, 1978). Los empleados valoran y hacen el mejor uso posible de los conocimientos y habilidades que cada miembro trae al equipo (Covey, 1989). Como resultado, existe un alto grado de colaboración. De lo contrario, cuando no existe sinergia, las personas se anulan unas a 
otras, rechazan la diversidad, se enfocan en las debilidades de los demás, no dan su máximo $\mathrm{y}$ el rencor prevalece entre los miembros del equipo.

\section{Dinamismo}

El grado en que las personas están involucradas en diversos proyectos, interesantes y significativos. Cuando la energía es intensa, los empleados saltan de una tarea completa a otra con continuado entusiasmo, compromiso, persistencia e intensidad. Los cambios constantes en procesos y procedimientos mantienen el ritmo veloz y estimulante en los lugares de trabajo (Ekvall, 1996; Isaksen, et al. 1999). En una organización con baja energia, las personas se vuelven apáticas y hasta defienden la rutina. Otros se aburren y se vuelven conformistas y la repetición prevalece en la organización.

\section{Espacio para las ideas}

El tiempo que se proporciona a las personas para proponer o desarrollar sus ideas (Ekvall, 1996; Isaksen et al., 1999; Amabile et al., 1999). Se brinda un tiempo, en forma habitual, para que grupos de empleados se reúnan con sus jefes o entre ellos para discutir propuestas y generar ideas para mejorar el trabajo, los productos, servicios, o procesos organizativos. Cuando el tiempo es insuficiente, no existe oportunidad para reflexionar porque el énfasis se encuentra en la producción en masa y el volumen a producir. Como consecuencia, las personas están estresadas porque la presión para producir es constante. No existe tiempo para relajarse.

\section{Fortalecimiento de confianza}

El grado en que la organización deliberadamente refuerza el sentido de seguridad y confianza en sus empleados para que fácilmente puedan proponer ideas y expresar sus opiniones (Anderson y West, 1996). La confian-za es alta y las personas valoran sus propias experiencias, fortalezas y habilidades cuando la organización implementa programas tales como capacitaciones, diálogos informales e intervenciones para abordar las preocupaciones de autoestima (Pierce et al., 1989), de una forma consistente, estratégica y consciente. Esto se ve reflejado en que los empleados demuestran poco miedo en recibir retroalimentaciones, son optimistas y resistentes. Cuando la organización ofrece poco para combatir las preocupaciones de autoestima, las personas limitan la expresión o defensa de sus ideas y puntos de vista (Osborn, 1963; Williams, 2002). Cuando sus ideas son objetadas, lo toman como algo personal, se sienten criticados, intimidados fácilmente $\mathrm{y}$ vulnerables al rechazo (Jones, 1987).

\section{Apoyo}

La forma en que se tratan las ideas y se cuida a las personas. Cuando existe apoyo, las ideas generalmente reciben buenas reacciones y se les da seguimiento (Ekvall, 1996; Isaksen et al., 1999), se proporcionan informes de los progresos y éstos son parte de un proceso continuo de respuesta a las ideas. Las personas perciben el apoyo emocional a sus ideas a través de reconocimiento, estímulo y al no sentir que están haciendo el ridiculo (Anderson y West, 1996; Amabile, 1996; Siegel y Kaemmerer, 1978). También perciben que a la empresa le importa el bienestar de sus empleados cuando se proporciona ayuda financiera, es decir cuando la gente recibe premios, galardones, dinero, alimentos, ayuda para transporte, o préstamos estudiantiles. Cuando falta el apoyo, las personas son reacias a escuchar ideas, las sugerencias son enfrentadas con criticismo, culpa, indiferencia y falta de seguimiento. Cuando a la empresa no le importa el bienestar de sus empleados, las personas se distraen y en vez de tener ideas relacionas con su trabajo, se preocupan más por pensar en cómo van a mantener a sus familias o a sí mismos.

\section{Creatividad deliberada}

La estructura de grupos pequeños activos, los sistemas analiticos y de prueba, y los procesos utilizados por la organización para impulsar a sus miembros a compartir y/o desarrollar ideas (Osborn, 1963; Basadur, 1987). Un ejemplo de esto son las sesiones de lluvia de ideas, administración de calidad total, comités y buzones de sugerencias. Cuando las medidas para manejar las ideas no son claras, las ideas no son canalizadas y se pierden o son pospuestas. Esto hace que las personas se sientan frustradas, se vuelven controversiales y dejen de presentar más ideas. Prevalece la sensación de que en la organización no vale la pena el esfuerzo. 


\section{Normas del manejo de influencia}

Las normas implícitas o explícitas que prevalecen en la organización para aceptar sólo a personas que pueden venderles ideas a quienes tienen el poder de implementarlas. Cuando hay normas positivas para el manejo de la influencia, se espera que las personas hablen del impacto de la idea en forma convincente. A las personas que son inquietas y persistentes se les presta más atención y la organización aprecia a las personas que superan objeciones. En un mal manejo de la influencia, la organización fácilmente descarta a las personas que no articulan el valor que una idea puede potencialmente traer a la organización. A veces las ideas se aceptan más por la insistencia y persuasión con que se someten que por el beneficio que potencialmente pueden traer a la organización. Así mismo, las personas no defienden vigorosamente sus propias ideas haciendo que las ideas que en otras circunstancias son ideas buenas se pierdan porque la persona que las somete no es un defensor eficaz de las mismas.

\section{Pertenencia}

El sentido significativo de conexión social y de lazos interpersonales que las personas sienten por otros miembros de la organización (Maslow, 1971; Schutz, 1994). Esto sucede cuando las personas sienten que son parte de la empresa, conocen a sus compañeros de trabajo y los pueden identificar por sus nombres e intereses. Además, las personas son incluidas en cuestiones de importancia para la empresa, se comprenden unas a otras en el trabajo, se sienten importantes y se identifican con el grupo. Cuando ocurre lo contrario, las personas son excluidas, existe el sentimiento de que las personas son percibidas como carentes de valor, insignificantes o vistas como un número.

\section{Envidia/Celos}

El grado en que las personas sienten resentimiento, envidia, celos o desventaja por los logros de los demás (Vai-Lam, 1995). Las personas comparan sus estructuras de poder, bienestar económico o estatus con las de los demás. Si una persona introduce una innovación o una idea que mejora su reputación, existe probabilidad de que se tomen represalias, se hagan sabotajes, robo de ideas o que se disfrute de los percances sufridos por el innovador. Cuando la envidia y los celos son minimos, las personas demuestran sincera gratitud, generosidad y admiración por el éxito de los demás.

\section{Sentido de equidad}

La imparcialidad percibida en los procedimientos y decisiones de la empresa (Lemons y Jones, 2001; Charness y Haruvy, 2000). Lo que se pone en el trabajo es razonablemente equitativo con lo que se obtiene del mismo. Lo que se da es el trabajo duro, lealtad, esfuerzo, determinación, tolerancia o compromiso con la empresa (Lemons y Jones, 2001; Charness y Haruvy, 2000). Lo que se obtiene de esos esfuerzos incluye la expectativa de un aumento de salario, capacitación y desarrollo, promociones, nuevas responsabilidades, elogios o viajes. Las personas sienten un alto nivel de satisfacción en el trabajo, identifican problemas y ofrecen soluciones. Las personas, además, consideran justas las decisiones y actuaciones de la empresa. Cuando no se percibe imparcialidad, las personas indirectamente disputan la falta de equidad en las decisiones y actuaciones de la empresa al no compartir sus ideas, quejándose, o haciendo sólo lo que se espera de ellas puesto que ven las decisiones de la empresa como egoístas. El favoritismo prevalece en este tipo de organización.

\section{Respuestas a las condiciones sociales, políticas y culturales (CSPC)}

Son las respuestas a eventos que se encuentran relativamente fuera del control de la organización, que desmotivan o alientan la creatividad e influyen en la habilidad de la organización para invertir en el desarrollo de ideas (Amabile, 1996). Tales eventos incluyen por ejemplo, crisis económicas, regulaciones impuestas por el gobierno, crimen, competencia global, deterioro de la confianza en la comunidad, guerra civil o un desastre natural. Cuando las organizaciones no responden constructivamente a una crisis o a una situa-ción, existe un sentido de incertidumbre y una especie de parálisis y las ideas no son de avanzada sino de sobrevivencia. Cuando ocurre lo contrario, las personas se sienten motivadas y desafiadas por las circunstancias por lo que siempre buscan formas de reinventarse. 


\section{Conclusiones}

Mathisen y Einarsen (2004) evaluaron cinco cuestionarios de clima creativo organizacional y describieron sus dimensiones respectivas. Los cuestionarios evaluados fueron diseñados en países desarrollados. Sin embargo, este estudio cualitativo produjo algunos temas que eran únicos para ciertas compañias colombianas tal como envidia/celos, sentido de equidad, CSPC, normas del manejo de influencia, la subcategoria soporte financiero, creatividad deliberada y pertenencia, temas no mencionados en los cuestionarios descritos en el artículo de Mathisen y Einarsen (2004).

Los resultados del estudio cualitativo pueden potencialmente convalidar el argumento de Brislin (1986) de que lo que se valora en una cultura puede no ser valorado en otra. Se puede mantener también que el costo de no considerar e investigar la influencia cultural podría ser grande. Por ejemplo, es probable que los investigadores diseminen información que no represente y comprenda inadecuadamente a grupos en organizaciones de otras culturas (Valencia, 2000). Ellos también pueden diagnosticar mal la manera como variables del tipo de la estructura familiar, el género, la edad, la religión, el nivel de bienestar económico y los valores culturales, influyen en la dinámica laboral, es decir, las intervenciones que dependen de la confrontación abierta, las cuales pueden producir en los miembros del grupo sentimientos de vergüenza y percepciones de insulto. Child (1981) explicaba que las brechas culturales tienen propensión a cerrarse sobre temas a nivel macro cuando las tecnologias organizacionales tales como la gerencia sobre calidad total son compartidas en todo el mundo, pero las diferencias únicas, tales como el comportamiento organizacional, amplían las brechas a nivel micro.

En muchos casos, los entrevistados mencionaron estos temas como obstáculos a la creatividad organizacional. A nivel nacional, el reporte del Global Competitiveness (20012002) mantiene que los departamentos de R\&D son limitados en Colombia. Sin embargo, Colombia no se debe confundir como una sociedad sin la capacidad de innovar. Herbig et al. (1998) discutieron que estos talentos están disponibles en cualquier sociedad pero si los talentos son o no son movilizados es primordialmente determinado por la cultura (Herbig et al., 1998). El nivel de la innovación que se genera en una cultura depende del grado de soporte que se proporciona a las iniciativas emprendedoras dentro de esa cultura (Herbig et al., 1998). Por ejemplo, Sull (2003) proporciona un caso pequeño de cómo tres negocios en países en vías de desarrollo superaron una carencia de recursos para sobresalir en creatividad e innovación al acercarse a los clientes y por lo tanto aprendiendo a solucionar problemas sin depender de nueva ciencia, innovando alrededor de tecnología, y buscando grandes ideas fuera del país. Es decir, se requiere de un acercamiento que sea sensible a las características culturales del país. Marin y Marin lo argumentan al manifestar que:

Es importante reiterar aquí que el desarrollo de instrumentos culturalmente apropiados, así como procedimientos culturalmente adecuados, protocolos de investigación e intervención, implican el ir más allá que la simple traducción o adaptación. En este sentido, no es suficiente obtener una buena traducción de un instrumento. Es también insuficiente solicitar a grupos étnicos la revisión y edición de un instrumento que ha estado previamente disponible para hacerlo más apropiado o aceptable para los implicados (1991, p. 66).

He aquí un ejemplo del previo argumento. La Tabla 2 compara y resalta algunas diferencias únicas que podrian potencialmente bloquear la exitosa implementación de tecnologías organizacionales al nivel micro. Como forma de demostrar estas diferencias, las categorias colombianas que emergieron en el presente estudio fueron comparadas con las dimensiones desarrolladas en Estados Unidos mediante el test Situational Outlook Questionnaire ${ }^{\circledR}$ (soQ). Hampden-Turner y Trompenaars observaron que las culturas nacionales no son diametralmente distintas las unas de las otras, pero a cambio "son imágenes que reflejan los valores de cada cual, e inversiones del orden y secuencia de ver y aprender" (2001, p. 1). La comparación en la Tabla 2 parece apoyar a Hampden y Trompenaars. El soQ fue traducido y diseñado para medir las percepciones de la caracterización de la vida de la organización en relación con el clima organizacional (Isaksen et al., 2000-2001). 
Tiempo para las ideas (grandes presupuestos para investigación y desarrollo). Enfoque intensivo en el capital.
Falta de tiempo (poco presupuesto para investigación y desarrollo; labor intensiva).
Lúdica/humor (informalidad).

Conflicto (comportamiento abierto).

Apoyo a las ideas (motivación intrínseca, orientada a la tarea).
Burocracia (formalidad).

Envidia/celos (comportamiento cubierto).
Apoyo socio-emocional y financiero (motivación extrínseca, orientada a las relaciones).

Debates (individualismo).

Sinergia (colectivismo).

Toma de riesgos (baja evasión de incertidumbre).

Rutina/Status quo (alta evasión de incertidumbre).

Retos e involucramiento (acercamientos orientados a la tarea).

Pertenencia (acercamientos orientados a la relación).

Libertad (minimo control, poco distanciamiento de poder).
Estilo de liderazgo autocrático (máximo control, alto distanciamiento de poder).
Confianza en las personas y en el sistema (universalismo).
Sentido de equidad: confianza en ciertas personas y sistemas (particularismo).
Apertura (la abundancia de recursos genera divergencia).
Influencia de normas administrativas (la falta de recursos genera convergencia). 
Estos resultados indican cinco puntos que se relacionan con el presente estudio. Primero, las estructuras pueden variar a través de los grupos. Segundo, hay aspectos del clima creativo en Colombia que necesitan ser entendidos y apreciados para facilitar la transferencia de la gerencia de sistemas como la solución creativa de problemas (Creative Problem Solving por su terminología en Inglés), TQM o planeación estratégica. Tercero, la traducción del cuestionario de clima creativo pudo haber omitido importantes ayudas o bloqueos a la creatividad en el lugar de trabajo. A cambio, incluye factores como la lúdica o satisfacción por el logro y retos, a expensas de factores como la pertenencia, envidia/celos, estilo de liderazgo (autocrático), apoyo al bienestar de los demás y la construcción de confianza, que son importantes en el caso colombiano según los resultados que arroja este estudio. Cuarto, las personas y organizaciones de los países en vías de desarrollo deben dejar de asumir que porque las teorias de comportamiento fueron desarrolladas en Estados Unidos son necesariamente buenas para ellos. Puede ser que las desarrolladas en Colombia sean más efectivas y apropiadas. Quinto, los datos del presente estudio sugieren que hay razones suficientes para justificar la necesidad de construir sistemas de medición nuevos y culturalmente más sensibles.

En conclusión, sí emergieron conceptos únicos para la muestra colombiana que potencialmente ameriten su inclusión en un cuestionario sobre el clima creativo. Por lo tanto, más conocimiento y más esfuerzo deliberado son necesarios para considerar la unicidad de la situación colombiana. 


\section{Referencias}

1. Abrahamson, M. (1983). Social Research Methods. New Jersey: Prentice Hall.

2. Amabile, T.M. (1996). Creativity in context. Colorado: Westview Press.

3. Amabile, T. M. y Gryskiewicz, S. S. (1988). Creative human resources in the RyD laboratory: How environment and personality affect innovation. En R.L. Kuhn (coordinador). Handbook for creative innovative managers. New York: McGraw-Hill.

4. Amabile, T. M., Burnside, R. M. \& Gryskiewicz, S. S. (1999). User's manual for assessing the climate for creativity: A survey from the center for creative leadership. North Carolina: Center for Creative Leadership.

5. Andersson, B. y Nilsson, S. (1964). Studies of the reliability and validity of the critical incident technique. Journal of Applied Psychology, 48(6), 398-403.

6. Anderson, N. R. y West, M. A. (1996). The Team Climate Inventory: Development of the $\mathrm{TCl}$ and its applications in teambuilding for innovativeness. European Journal of Work and Organizational Psychology, 5, 53-66.

7. Andriopoulos, C. y Dawson, P. (2009). Managing change, creativity and innovation. London: Sage.

8. Arksey, H. y Knight, P. (1999). Interviewing for social scientists. London: Sage.

9. Basadur, M. S. (1987). Needed research in creativity for business and industrial applications. En S. G. Isaksen (coordinador). Frontiers of creativity research: Beyond the basics. New York: Bearly.

10. Berg, B. L. (2001). Qualitative research methods for the social sciences, $4^{\text {th }}$ ed. Massachusetts: Allyn and Bacon.
11. Bridges, W. (1991). Managing transitions: making the most of change. Massachusetts: Addison-Wesley Publishing Company.

12. Brislin, R. W. (1986). The wording and translation of research instruments. En W. J. Lonner y J. W. Berry (coordinador.) Field methods in crosscultural research. California: Sage.

13. Burnside, R. M., Amabile, T. M. \& Gryskiewicz, S.S. (1988). Assessing organizational climates for creativity and innovation: methodological review of large company audits. En I. Yuji y R. L. Kuhn (coordinador). New directions in creative and innovative management: Bridging theory and practice. Cambridge. Massachusetts: Ballinger Publishing Company.

14. Cabra, J. F. (2006). An exploratory examination of creative climate expectations among Colombian managers, supervisors and frontline employees and subsequent development of a measure to assess creative climate. An unpublished doctoral thesis, University of Manchester, Manchester, U.K.

15. Charness, G. y Haruvy, E. (2000). Self-serving biases: evidence from a simulated labour relationship. Journal of Managerial Psychology, 15(7), 655-667.

16. Child, J. (1981). Culture, contingency, and capitalism in the cross-national study of organizations. En L. L. Cummings y B. M. Staw (coordinador). Research in organizational behavior. Connecticut: JAI Press.

17. Covey, S. R. (1989). The 7 habits of highly effective people: powerful lessons in personal change. New York: Fireside.

18. Ekvall, G. (1996). Organizational climate for creativity and innovation. European Journal of Work and Organizational Psychology, 5(1), 105-123.

19. Ekvall, G., Avrvonen, J. \& Waldenström-Lindblad, I. (1983). Creative organizational climate: construction and validation of a measuring instrument (Report 2). Sweden: FA rådet. The Swedish council for management and organizational behavior. 
20. Firestien, R. L. (2004). Leading on the creative edge: Gaining competitive advantage through the power of Creative Problem Solving. Williamsville, NY: Innovation Resources.

21. Global Competitiveness Report (2001-2002). World Economic Forum and Harvard University. New York, Oxford University Press.

22. Hampden-Turner, C. y Trompenaars, F. (2000). Building cross-cultural competence: How to create wealth from conflicting values. Connecticut: Yale University Press.

23. Henry, J. (2001). Creativity and perception in management. London: Sage.

24. Herbig, P. y Dunphy, S. (1998). Culture and innovation. Cross-Cultural Management, 5(4), 13-23.

25. IBM Institute for Business Value (2010). Cultivating organizational creativity in an age of complexity. IBM Global Chief Resource Officer Study.

26. Isaksen, S. G., Lauer, K. J., Ekvall, G. \& Britz (2000-2001). Perceptions of the best and worst climates for creativity: Preliminary validation evidence for the situational outlook questionnaire. Creativity Research Journal, 13 (2), 171-184.

27. Isaksen, S. G., Lauer, K. J. \& Ekvall, G. (1999). Situational outlook questionnaire: A measure of the climate for creativity and change. Psychological Reports, 85, 665-674.

28. Jones, L. (1987). Barriers to effective problem solving. Creativity and Innovation Network, 10 (2). Manchester Business School, Manchester.

29. Jorgensen, J. J., Hafsi, T. \& Kiggundu, M. N. (1986). Towards a market imperfections theory of organizational structure in developing countries. Journal of Management Studies, 23(4), 417-442.

30. Kanter, R. M. (1984). The change masters. New York: Touchtone.
31. Lee, B. (1997). The Power Principle. New York: Simon Schuster.

32. Lemons, M. A. y Jones, C. A. (2001) Procedural justice in promotion decisions: using perceptions of fairness to build employee commitment. Journal of Managerial Psychology. 16(4), 268-280.

33. Marin, G. y Marin, B. (1991). Research with Hispanic populations. Thousand Oaks, California: Sage.

34. Maslow, A. (1971). The farther reaches of human nature. New York: Viking.

35. Matisen, G. E. y Einarsen, S. (2004). A review of instruments assessing creative and innovative environments within organizations. Creativity Research Journal, 16(1), 119-140.

36. Miles, M. B. y Huberman, A. M. (1994). Qualitative data analysis: an expanded sourcebook, $2^{\text {nd }}$ ed. California: Sage Publications.

37. Osborn, A. F. (1963). Applied Imagination, $3^{\text {rd }}$ ed. New York: Scribner's Sons.

38. Pierce, J. L., Gardner, D. G., Cummings, L. L. \& Dunham, R. B. (1989). Organization-based self-esteem: Construct definition, measurement, and validation. Academy of Management Journal, 32(3), 622-648.

39. Rhodes, M. (1961). An analysis of creativity. Phi Delta Kappan, 42, 305-310.

40. Schneider, S. (1988). National vs. corporate culture: implications for human resource management. Human Resource Management, 27, 231-246.

41. Schutz, W. (1994). The human element. California: Jossey-Bass.

42. Siegel, S. M. y Kaemmerer, W. F. (1978). Measuring the perceived support for innovation in organizations. Journal of Applied Psychology 63: 553-562. 
43. Strauss, A. L. y Corbin, J. (1990). Basic qualitative research: Grounded theory procedures and techniques. London: Sage Publications.

44. Sull, D. N. (November-December, 2003). Innovating around obstacles. What developingworld companies teach us about innovation. Strategy \& Innovation, 3-6.

45. Thomas, K.W.y Kilmann, R. H. (1974). ThomasKilmann conflict mode instrument. New York: Xicom.

46. Vai-Lam, M. (1995) The economics of envy. Journal of Economic Behavior and Organization. 26, 311-336.

47. Valencia, E.Y. (2000). Value orientations in Colombia: variations in age, gender, and SES: An empirical study. Unpublished doctoral dissertation. The Chicago School of Professional Psychology, Chicago, Illinois.

48. Van Gundy, A. (1987). Organizational creativity and innovation. En S. G. Isaksen (coordinador). Frontiers of creativity research: Beyond the basics. New York: Bearly.

49. Williams, S. D. (2002). Self-esteem and the self-censorship of creative ideas. Personnel Review, 31(4), 495-503. 